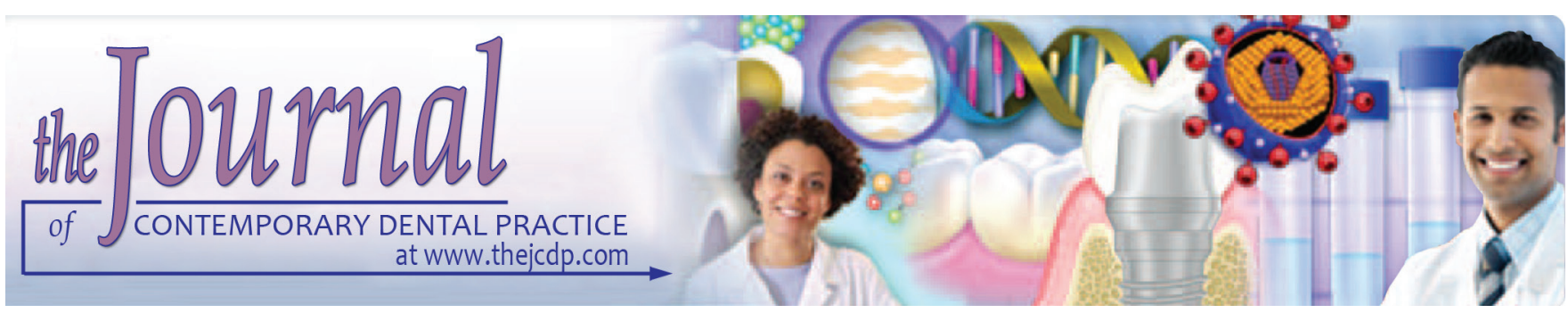

\title{
Adjunctive Systemic Antimicrobial Therapy vs Asepsis in Conjunction with Guided Tissue Regeneration: A Randomized, Controlled Clinical Trial
}

\author{
Mahmoud Abu-Ta'a
}

\section{ABSTRACT}

Aim: This randomized clinical trial compares the usefulness of adjunctive antibiotics, while strict asepsis was followed during periodontal surgery involving guided tissue regeneration.

Materials and methods: Two groups of 20 consecutive patients each with advanced periodontal disease were randomly assigned to treatment. They displayed one angular defect each with an intrabony component $\geq 3 \mathrm{~mm}$, probing pocket depth and probing attachment level (PAL) $\geq 7 \mathrm{~mm}$. Test group included 13 males, mean age 60 years, treated with enamel matrix derivative (EMD) and demineralized freeze-dried bone allograft with modified papilla preservation technique, received oral amoxicillin $1 \mathrm{gm}, 1$ hour preoperatively and $2 \mathrm{gm}$ for 2 days postoperatively. Control group included 10 males, mean age 57 years, treated with EMD and demineralized freeze-dried bone allograft with modified papilla preservation technique, received no antibiotics.

Outcome measures were clinical attachment level (CAL) gain, residual periodontal pocket depth (res. PD), gingival recession (GR), bleeding on probing (BOP), adverse events and postoperative complications.

Patients were followed up to 12 months after periodontal surgery involving guided tissue regeneration.

Results: There were no significant differences between both groups for CAL gain, res. PD, GR, BOP nor other clinical parameters, though patients' subjective perception of postoperative discomfort was significantly smaller in the group receiving antibiotics.

Conclusion: Antibiotics do not provide significant advantages concerning clinical periodontal parameters nor concerning postoperative infections in case of proper asepsis. It does, on the contrary, reduce postoperative discomfort.

Department of Oral Surgery and Periodontology, Arab American University, Jenin, Palestine

Corresponding Author: Mahmoud Abu-Ta'a, Assistant Professor, Department of Oral Surgery and Periodontology, Arab American University Jenin, Palestine, Phone: 00972547380763 e-mail: mahmoud.abutaa@aauj.edu
Clinical significance: Regarding the results of this study, adjunctive systemic antibiotics in combination with guided tissue regeneration may be useful in reducing postoperative discomfort but may not be helpful for improving periodontal regeneration outcomes.

Keywords: Angular defect, Antibiotic prophylaxis, Guided tissue regeneration.

How to cite this article: Abu-Ta'a M. Adjunctive Systemic Antimicrobial Therapy vs Asepsis in Conjunction with Guided Tissue Regeneration: A Randomized, Controlled Clinical Trial. J Contemp Dent Pract 2016;17(1):3-6.

Source of support: Nil

Conflict of interest: None

\section{INTRODUCTION}

The periodontal tissues, including alveolar bone, periodontal ligament and root cementum, play an important role to support the normal function of a tooth. Unfortunately, inflammation of these supporting tissues, periodontitis, can cause periodontal breakdown and subsequent tooth loss, if untreated. ${ }^{1}$ In addition to resolving the inflammatory process and its negative consequences, the ultimate goal of periodontal therapy is to increase the periodontal attachment of a severely compromised tooth through various regenerative therapies, such as guided tissue regeneration (GTR). ${ }^{1}$

Periodontal surgical procedures including GTR procedures carry with them an inherent risk of developing complications such as infections. ${ }^{2}$ It is especially important to avoid perioperative infection of the wound during surgery, when foreign bodies are implanted. ${ }^{3}$ Indeed, biofilm-shielded microbial colonization on the surface of the latter is much more resistant to antimicrobials than their planctonic form. ${ }^{4}$

In spite of the fact that dentists, oral surgeons and periodontists often prescribe antibiotics routinely following oral surgical procedures, the validity of such 
tradition remains questionable. The side effects of antibiotic therapy are well documented and can be serious, including anaphylaxis and the possibility for development of resistant microbial strains. ${ }^{5}$

It has been previously shown that antibiotic prophylaxis offers no advantage in preventing postoperative infections or affecting periodontal surgery outcomes. ${ }^{6}$ The rate of infections following periodontal surgery, when no antibiotics were used, ranges from less than 1 to $4.4 \%{ }^{7}$

Several sources of infection during surgery in the oral cavity have been identified: instruments, the hands of surgeon and assistants, the air of the operatory room (OR), patients' nostrils and saliva, and the perioral skin. ${ }^{8}$ During intraoral surgery, reduction of salivary flow can be achieved by atropine and of the microbial flora by preoperative rinsing with chlorhexidine. The supine position of the patient and the use of two independent suction tips (one for the mouth and one only for the wound) can further decrease the chances of wound contamination. ${ }^{8}$ The use of a meshed nose guard prevents contact with the highly contaminated nares, while it was demonstrated that the expired air does not contain more bacteria than the surrounding air of the OR. ${ }^{8}$

The aim of this randomized, controlled clinical trial was to compare the usefulness of pre- and postoperative antibiotics, while strict asepsis was followed during periodontal surgery involving GTR.

\section{MATERIALS AND METHODS}

This prospective, randomized, controlled clinical trial involved 40 consecutive patients with one angular defect each displaying an intrabony component $\geq 3 \mathrm{~mm}$, probing pocket depth (PPD) and probing attachment level (PAL) $\geq 7 \mathrm{~mm}$. Three months following the initial, cause-related phase of periodontal therapy and at a reevaluation visit, all patients presented full mouth plaque scores of $(9.2 \pm 1.7) \%$ and full mouth bleeding scores $(7.6 \pm$ $2.8) \%$. Patients were randomly assigned into one of two groups (test and control) of 20 patients each using random sampling with masking of the person performing the randomization and after each signing an informed consent. Antibiotics group (test) included 13 men, mean age 60 years, treated with enamel matrix derivative (EMD); Straumann AG, Basel, Switzerland following demineralization with EDTA (24\% for 2 minutes) and demineralized freeze-dried bone allograft (DFDBA) with modified papilla preservation technique; received oral amoxicillin $1 \mathrm{gm}, 1$ hour preoperatively and $2 \mathrm{gm}$ for 2 days postoperatively.

The nonantibiotics group (control) consisted of 20 patients: 10 men and 10 women, mean age 57 years, range 26 to 88 years. In this group, patients were treated with EMD; Straumann AG, Basel, Switzerland following demineralization with EDTA (24\% for 2 minutes) and DFDBA with modified papilla preservation technique; received no antibiotics.

The exclusion criteria for the study were allergy to penicillin, need for endocarditis prophylaxis, any systemic or local immunodeficiency, uncontrolled diabetes mellitus, smoking or previous radiation therapy in the head and neck area. Such patients would systematically receive prophylactic antibiotics and were not considered for randomization.

All patients were instructed to rinse with chlorhexidine digluconate $(0.12 \%$ solution without alcohol) for 1 minute just before surgery. Postoperatively, all patients were rinsed with the same agent twice a day for 1 minute up to the follow-up visit (stitch removal), which was 7 to 10 days later. After stitch removal, all patients followed a tight infection control protocol that extended for 5 weeks, including weekly professional tooth cleaning consisting of supragingival prophylaxis with a rubber cup and chlorhexidine gel. They were advised not to perform mechanical oral hygiene measures and as much as possible not to chew on the treated sides for 5 weeks. After this period, all patients were re-instructed to resume mechanical oral hygiene gradually, including interdental cleaning, and to discontinue chlorhexidine. Patients were enrolled in a periodontal care program on a monthly basis until 1 year. Probing or deep scaling in the treated areas was generally avoided before the 1-year follow-up visit.

Both the surgical team and the patients were blinded to the groups. Outcome measures were clinical attachment level (CAL) gain, residual periodontal pocket depth (res. $\mathrm{PD})$, gingival recession (GR), bleeding on probing (BOP), adverse events and postoperative complications.

Patients were followed up to 12 months after periodontal surgery involving GTR.

Measures of asepsis and prevention against infection from the oral cavity included the use of sterile drapes around the patient's mouth, head and a large sterile drape over the supine body of the patient as usually done during periodontal surgery in addition to the use of two suction tips (one for the mouth and one only for the wound). All 40 patients were seen at a follow-up visit (stitch removal appointment) 7 to 10 days after periodontal surgery. At the end of this appointment, both the periodontist, who removed the stitches, and the patient were asked to fill in a 10 -cm visual analogue score (10 cm VAS score) consisting of five questions, in Arabic, to evaluate symptoms of infection/inflammation, which were spontaneous and/ or evoked pain, erythema, swelling and pus. ${ }^{6}$ A score of 10 meant no pain, no swelling, etc. 


\section{Statistical Analysis}

Data were statistically analyzed by means of STATWING software (2012) for windows. To evaluate the differences in means between both test and control groups, a t test for independent unpaired variables was used. The $p$ value was set at the 0.05 level to detect significance. The null hypothesis was the absence of significant differences between groups.

\section{RESULTS}

All 40 patients randomly allocated to one of the groups completed the study and all of them were controlled at the follow-up visits by a periodontist (Flow Chart 1). All participants were included in the statistical analyses (test, $\mathrm{n}=20$; control, $\mathrm{n}=20$ ). Guided tissue regeneration for intrabony defects resulted in large CAL gains and minimal GRs at 1 year. The average CAL gain observed at 1 year was $4.3 \pm 1.1 \mathrm{~mm}$ for the test group and $4.1 \pm 1.4$ $\mathrm{mm}$ for the control group. In addition, the average GR was almost the same for both groups, $0.4 \pm 0.7 \mathrm{~mm}$. Shallow residual pockets (res. PD) were consistently found at 1 year for patients in both groups; average res. PD for the antibiotics groups was $3.4 \pm 1.2$ vs $3.7 \pm 1.3 \mathrm{~mm}$ for the no-antibiotics group.
Other clinical parameters, namely plaque scores, BOP and gingivitis scores, were similar in the test and control sites 12 months following GTR.

Finally, there were no significant differences between both groups regarding the assessment of symptoms of infection/inflammation by the periodontist at the time of suture removal (VAS scores), although erythema and swelling occurrence was slightly more in the control group.

\section{DISCUSSION}

The present randomized controlled clinical study demonstrated that the differences between the two treatment modalities did not reach statistical significance, thus indicating no added benefit of combining systemic amoxicillin with periodontal surgery involving GTR, although patients' subjective perception of postoperative discomfort was significantly smaller in the group receiving antibiotics.

Apart from erythema and swelling, more severe postoperative complications such as suppuration, sloughing, perforations of the flap, and postoperative pain have not been reported in either of the groups confirming the fact that there was no added benefit of perioperative

Flow Chart 1: Patients' flow throughout the phases of the study

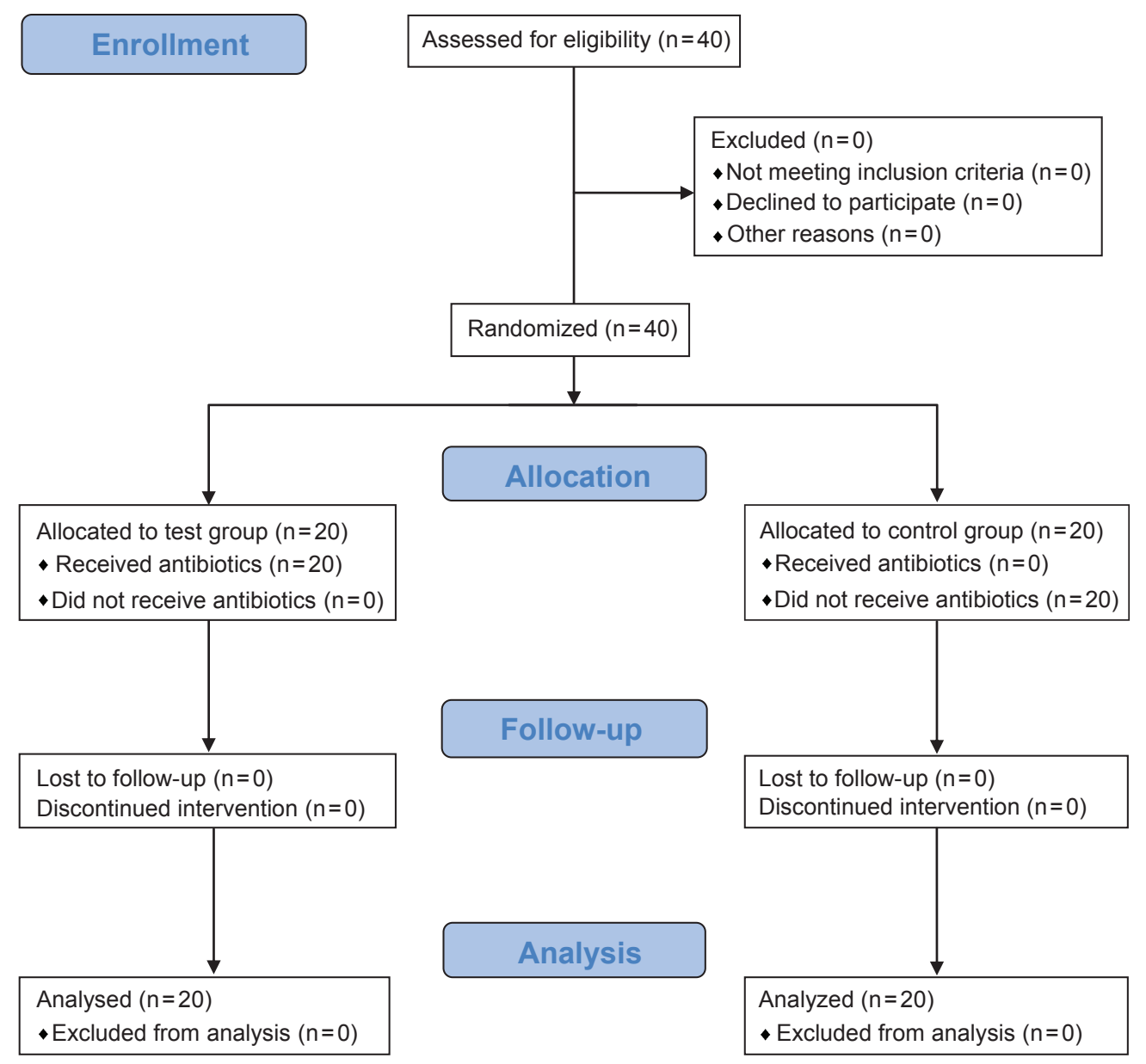


antibiotics throughout this study on periodontal healing after GTR. It should be emphasized that a strict policy of asepsis was followed in this clinical study.

\section{CONCLUSION}

Antibiotics do not provide significant advantage regarding clinical periodontal parameters nor concerning postoperative infections in case of proper asepsis during routine periodontal surgery involving GTR. It might, on the contrary, reduce postoperative discomfort.

The results of this clinical trial suggest that wellmaintained high standards of asepsis might be preferred over perioperative antibiotics, particularly when estimating the benefits and risks of antibiotics confirming the fact that antibiotics should not be used as a cover-up for inappropriate asepsis techniques.

\section{REFERENCES}

1. Villar CC, Cochran DL. Regeneration of periodontal tissues: guided tissue regeneration. Dent Clin North Am 2010 Jan; 54(1):73-92

2. Salvi M, Chelo C, Caputo F, Conte M, Fontana C, Peddis $\mathrm{G}$, Velluti $\mathrm{C}$. Are surgical scrubbing and pre-operative disinfection of the skin in orthopaedic surgery reliable? Knee Surg Sports Traumatol Arthrosc 2006 Jan;14(1):27-31.

3. Haanaes, H.R. Implants and infections with special reference to oral bacteria. J Clin Periodontol 1990 Aug;17(7 Pt 2):516-524.

4. Engemann JJ, Carmeli Y, Cosgrove SE, Fowler VG, Bronstein MZ, Trivette SL, Briggs JP, Sexton DJ, Kaye KS. Adverse clinical and economic outcomes attributable to methicillin resistance among patients with Staphylococcus aureus surgical site infection. Clin Infect Dis 2003 Mar1;36(5):592-598.

5. Dent CD, Olson JW, Farish SE, Bellome J, Casino AJ, Morris HF, Ochi $\mathrm{S}$. The influence of preoperative antibiotics on success of endosseous implants up to and including stage II surgery: a study of 2,641 implants. J Oral Maxillofac Surg 1997 Dec;55 (12 Suppl 5):19-24.

6. Abu-Ta'a M, Quirynen M, Teughels W, van Steenberghe D. Asepsis during periodontal surgery involving oral implants and the usefulness of peri-operative antibiotics: a prospective, randomized, controlled clinical trial. J Clin Periodontol 2008 Jan;35(1):58-63.

7. Pack PD, Haber J. The incidence of clinical infection after periodontal surgery. A retrospective study. J Periodontol 1983 Jul;54(7):441-443.

8. van Steenberghe D, Yoshida K, Papaioannou W, Bollen CM, Reybrouck G, Quirynen M. Complete nose coverage to prevent airborne contamination via nostrils is unnecessary. Clin Oral Implants Res 1997 Dec;8(6):512-516. 